\title{
Durvalumab monotherapy as a third-line treatment for extensive-stage small-cell lung cancer: a case report
}

\author{
Qinfei Zhou, ${ }^{1,2 \#}$, Jiazheng Zhao, ${ }^{2,3 \#}$, Jie Wang ${ }^{4}$, Guanai Bao ${ }^{2,5}$, Li-Yan Gong ${ }^{1,2}$ \\ ${ }^{1}$ Department of Rare and Head and Neck Oncology, Cancer Hospital of the University of Chinese Academy of Sciences (Zhejiang Cancer Hospital), \\ Hangzhou, China; ${ }^{2}$ Institute of Cancer and Basic Medicine (IBMC), Chinese Academy of Sciences, Hangzhou, China; ${ }^{3}$ Department of Head and \\ Neck Surgery, Cancer Hospital of the University of Chinese Academy of Sciences (Zhejiang Cancer Hospital), Hangzhou, China; ${ }^{4}$ Department of \\ Oncology, Jinhua Guangfu Cancer Hospital, Jinhua, China; ${ }^{5}$ Department of Integrated Chinese Traditional Medicine and Western Medicine, Cancer \\ Hospital of the University of Chinese Academy of Sciences (Zhejiang Cancer Hospital), Hangzhou, China \\ \#These authors contributed to the work equally and should be regarded as co-first authors. \\ Correspondence to: Li-Yan Gong. Department of Rare and Head and Neck Oncology, Cancer Hospital of the University of Chinese Academy of \\ Sciences (Zhejiang Cancer Hospital), Hangzhou, China; Institute of Cancer and Basic Medicine (IBMC), Chinese Academy of Sciences, No. 1 East \\ Banshan Road, Gongshu District, Hangzhou, China. Email: susang409@hotmail.com.
}

\begin{abstract}
The global incidence and mortality rates of lung cancer are the highest of any cancer. Smallcell lung cancer (SCLC) is an undifferentiated carcinoma which accounts for $15-20 \%$ of all lung cancers. Compared with the other major lung cancer type, non-small cell lung cancer, SCLC exhibits worse biological behavior, has a higher degree of malignancy, and develops more rapidly. The majority of SCLC present with extensive-stage disease, and the prognosis for these patients remains poor. Recently, immunotherapy has been demonstrated clinical activity in extensive-stage SCLC (ES-SCLC); however, the efficacy and safety of immunotherapy in ES-SCLC needs further confirmation. Durvalumab, a selective, high-affinity human IgG1 monoclonal antibody that blocks PD-L1 binding to PD-1 and CD80, showed durable clinical activity and a manageable safety profile in patients with pretreated ES-SCLC as a first-line treatment. Here, we report the case of an ES-SCLC patient who achieved complete remission (CR) of local lesions after receiving durvalumab monotherapy as a third-line treatment, experiencing no obvious immune-related side effects, such as rash, diarrhea, fatigue, myelosuppression, or thyroid dysfunction. No immune-related pulmonary or hepatorenal toxicities occurred. The case suggests that immunotherapy can be selected for third-line or multi-line treatment of ES-SCLC, and anti-PD-L1 antibody may be the better choice for patients who have poor performance status (PS) scores.
\end{abstract}

Keywords: Small-cell lung cancer (SCLC); immunotherapy; durvalumab; third-line treatment; case report

Submitted May 26, 2020. Accepted for publication Jul 09, 2020.

doi: 10.21037/apm-20-1244

View this article at: http://dx.doi.org/10.21037/apm-20-1244

\section{Introduction}

The global incidence and mortality rates of lung cancer are the highest of any cancer. Small-cell lung cancer (SCLC) is an undifferentiated carcinoma which accounts for $15-20 \%$ of all lung cancers. Compared with the other major lung cancer type, non-small cell lung cancer, SCLC exhibits worse biological behavior, has a higher degree of malignancy, and develops more rapidly. Extensive- stage SCLC (ES-SCLC) has been reported to make up $60-70 \%$ of all SCLC cases (1). For patients with ESSCLC, chemotherapy has been the main treatment for more than 30 years. The standard first-line treatment has consisted of etoposide plus either cisplatin or carboplatin (platinum-etoposide), with few alternatives, despite primary chemotherapy with platinum-etoposide having a high response rate of up to $78 \%(2,3)$, most patients relapse 
within 6 months of the completion of initial treatment and the median overall survival (mOS) is a meager 10 months $(2,4,5)$. Dozens of phase III trials have failed to improve survival over standard platinum plus etoposide. Topotecan is the only drug approved for use as a second-line treatment of SCLC by the United States Food and Drug Administration. However, the median duration of remission (DOR) of topotecan is only 3.3 months, and the efficacy is not satisfactory, with toxic reactions including severe neutropenia and anemia. Despite this being actively explored in various clinical trials, the improvement in the survival of patients with ES-SCLC has been minimal for decades, and there is a desperate need for new drugs of clinical value to be developed.

However, resent results have met with long-overdue success. Immune checkpoint inhibitor is a new anti-tumor drug that regulate the normal activity of immune cells by blocking the interaction of inhibitory receptors and related ligands expressed on $\mathrm{T}$ cells, to achieve an antitumor effect. In recent years, immunotherapy targeting the programmed cell death 1 (PD-1) and programmed cell death ligand 1 (PD-L1) pathway has demonstrated clinical activity for patients with ES-SCLC, including as a first-line treatment. Two recent phase III trials had shown an improvement in overall survival (OS) with concurrent immunotherapy and chemotherapy. IMpower 133 was a global phase I/III, double-blind, randomized, placebocontrolled study. In IMpower 133, the addition of the anti-PD-L1 antibody atezolizumab to carboplatin plus etoposide significantly improved both progression-free survival (PFS) and OS (6). This was the first trial in over 30 years to improve survival. Another phase III trial was CASPIAN, also intended to prove the efficacy and safety with concurrent immunotherapy and chemotherapy. The result showed that the addition of another anti-PD-L1 antibody durvalumab plus platinum-etoposide significantly demonstrated improved OS in patients with ES-SCLC as a first-line treatment (7). While there is clearly a need to further improve outcomes, the improvement in survival with the addition of atezolizumab or durvalumab to platinum-etoposide chemotherapy is a major advance.

Durvalumab, a selective, high-affinity human IgG1 monoclonal antibody that blocks PD-L1 binding to PD-1 and CD80 (8), is indicated for the treatment of patients with unresectable, stage 3 non-small cell lung cancer following platinum based chemoradiotherapy $(9,10)$. In early-phase clinical trials, durvalumab, both as monotherapy and in combination with the anti-cytotoxic $\mathrm{T}$ lymphocyte- associated antigen- 4 antibody, tremelimumab, showed durable clinical activity and a manageable safety profile in patients with pretreated ES-SCLC, including those with relapsed or refractory disease (11-13).

Considering the results of current clinical research, the following case is reported as a real event encountered in our clinical work. We present the following case in accordance with the CARE reporting checklist (available at http:// dx.doi.org/10.21037/apm-20-1244).

\section{Case presentation}

A 66-year-old woman with a past medical history of hyperlipidemia, first presented at our hospital on August 21, 2017, with a cough and chest tightness that had lasted more than a month. The patient underwent tracheoscopy, and she was diagnosed with SCLC and confirmed by pathology. On August 29, the patient underwent positron emission tomography-computed tomography (PET-CT) examination. A right hilar malignant tumor (lung cancer) accompanied by right upper lobe obstructive atelectasis was detected, along with mediastinum and right clavicular lymph node metastasis. Consequently, the patient was clinically diagnosed with right lung localized SCLC (right hilar, mediastinum, and right supraclavicular lymph node metastasis). Between September 4, 2017 and February 6, 2018 , the patient received five cycles of chemotherapy with platinum-etoposide and chest radiotherapy. After the first stage of treatment, a chest CT reexamination was conducted. The pulmonary lesions were evaluated, and the patient was judged to have achieved partial remission (PR) (Figure 1).

After a 4-month remission, the patient visited the hospital again on June 9, 2018, after experiencing dizziness for two weeks. Brain magnetic resonance imaging (MRI) indicated brain metastasis accompanied by supraclavicular lymph node metastasis. The pulmonary lesions were stable. The clinical diagnosis was extensive-stage SCLC. The patient underwent whole brain radiotherapy (3,000 cgy/10 F), which was completed in June, 2018, followed by four cycles of chemotherapy with irinotecan-carboplatin beginning in July, 2018. In October 2018, the patient was reexamined by brain MRI and chest CT. The pulmonary lesions were stable, and the brain lesions had shrunk; partial remission was confirmed (Figure 2). The patient received maintenance chemotherapy with irinotecan until April, 2019.

On August, 2019, the patient was reexamined with B-mode ultrasound, and the presence of several hypoechoic 

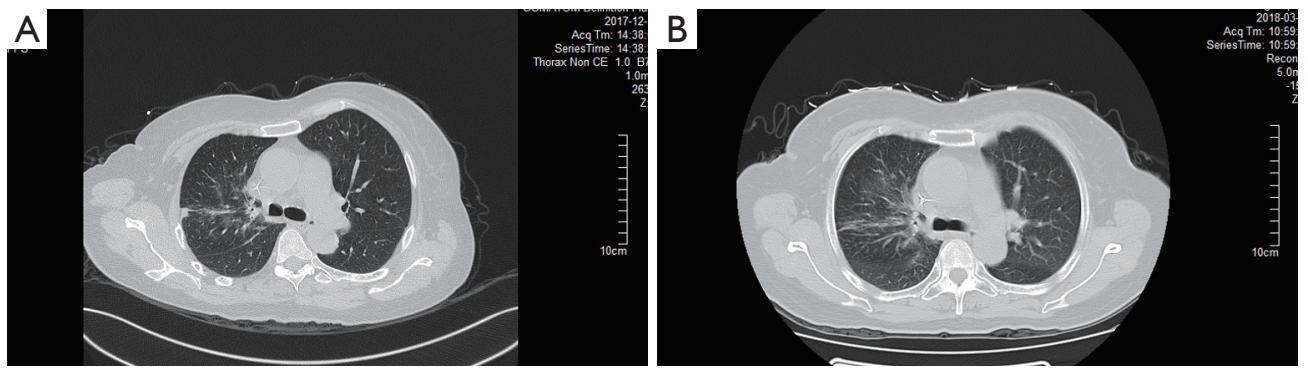

Figure 1 The chest CT images of the patient at different stages. (A) The patient was reexamined on December 2017 after five cycles of chemotherapy; (B) the patient was reexamined on March 2018 after chest radiotherapy.
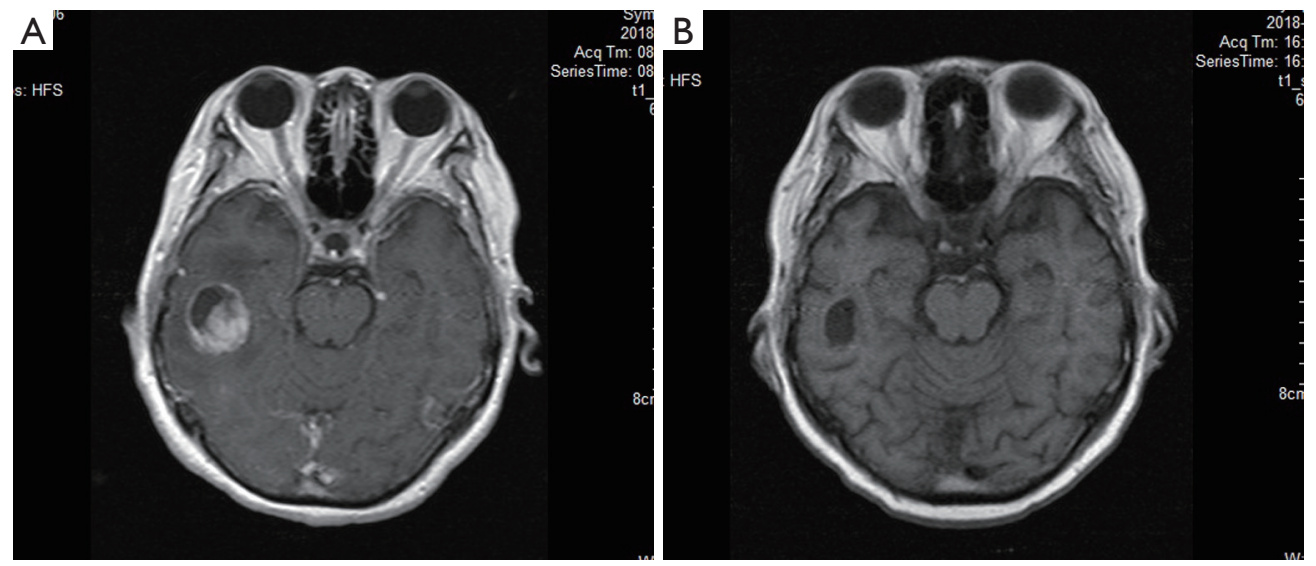

Figure 2 The brain MR images of the patient at different stages. (A) Reexamination on June, 2018, before the brain radiotherapy; (B) reexamination on October, 2018, after brain radiotherapy and four cycles of chemotherapy.

nodules was indicated on the right lower neck and the right clavicle, with the largest measuring approximately $45 \mathrm{~mm}$ $\times 21 \mathrm{~mm} \times 28 \mathrm{~mm}$ (Figure 3). The pulmonary and brain lesions were stable. Because of the new cervical metastasis, the patient needed third-line treatment. Considering the patient's poor constitution, with a WHO performance status score of 2 and anemia, durvalumab plus platinumetoposide with a dose reduction was the chosen treatment. After an improvement was observed in the patient's routine blood, biochemical, thyroid function, and other test results, the first cycle of durvalumab plus platinum-etoposide (durvalumab 1,500 $\mathrm{mg}$ day 1 ; etoposide $120 \mathrm{mg}$ day $1-3$; platinum $30 \mathrm{mg}$ day 1-3) was completed on August 28, 2019. However, after the first cycle of treatment, the patient developed serious complications: upper gastrointestinal hemorrhage appeared after the patient had returned home, with a bleeding volume reaching $200 \mathrm{~mL}(9.19$ routine blood: white blood cell (WBC) count $2.4 \times 10^{9} / \mathrm{L}$; hemoglobin level: $6.2 \mathrm{~g} / \mathrm{dL}$; platelet count: $\left.48 \times 10^{9} / \mathrm{L}\right)$. As well as having severe anemia, the patient also had grade III myelosuppression, accompanied by obvious asthenia. After one month of symptomatic treatment in the local hospital, the patient's condition gradually improved.

On October 23, 2019, the patient returned to our department for reexamination (10.23 routine blood: WBC: $4.2 \times 10^{9} / \mathrm{L}$; hemoglobin: $11.1 \mathrm{~g} / \mathrm{dL}$; platelet count: $\left.100 \times 10^{9} / \mathrm{L}\right)$. Gastroscopy suggested chronic atrophic gastritis, and B-ultrasound showed that the right cervical lymph nodes were significantly reduced, with the largest measuring $24 \mathrm{~mm} \times 11 \mathrm{~mm}$ (right neck) and $22 \mathrm{~mm} \times 18 \mathrm{~mm}$ (right supraclavicle). Considering that the patient had previously experienced serious complications after the first cycle of treatment, and we believed these complications due to chemotherapy, so from the second cycle onwards, chemotherapy was cancelled, leaving durvalumab monotherapy $(1,500 \mathrm{mg} \mathrm{d} 1 / \mathrm{d} 3 \mathrm{w})$. After three cycles of immunotherapy, brain MRI and chest CT confirmed that the lesions in the brain and lung were stable. After four 

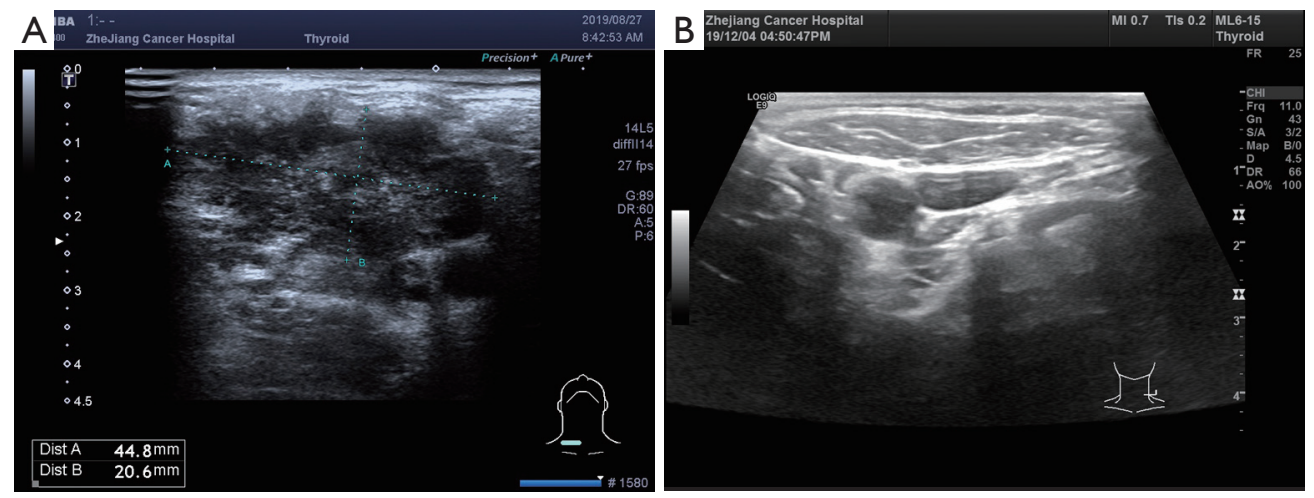

Figure 3 The ultrasound images of the patient at different stages. (A) Before immunotherapy, the largest cervical lymph node measured about $45 \mathrm{~mm} \times 21 \mathrm{~mm} \times 28 \mathrm{~mm}$; (B) after four cycles of immunotherapy, no abnormal lymph nodes were found.
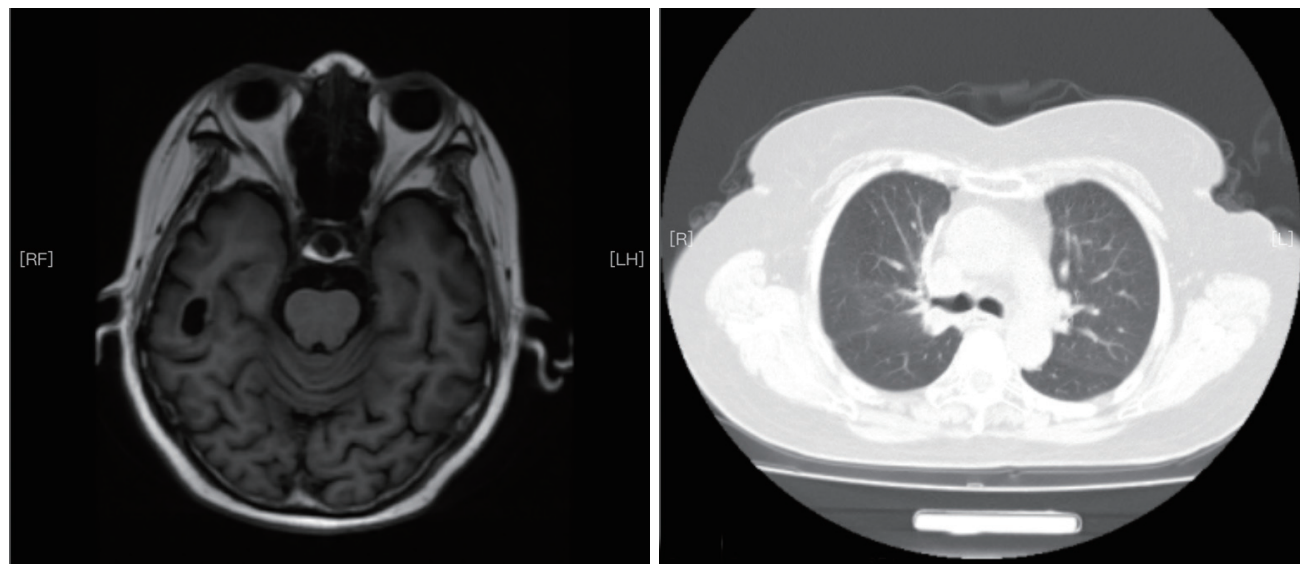

Figure 4 During reexamination on May, 2020, the lesions in the patient's brain and lung were stable.

cycles of immunotherapy, no abnormal lymph nodes were found in B-ultrasound of the neck (Figure 3). Cervical lesions achieved complete remission (CR). To date, the case had complete 12 cycles of immunotherapy, experiencing no obvious immune-related side effects, such as rash, diarrhea, fatigue, myelosuppression, or thyroid dysfunction. No immune-related pulmonary or hepatorenal toxicities occurred. The last reexamination of the patient was conducted in May, 2020. The brain and pulmonary lesions were shown to be stable (Figure 4), and the cervical lesions were in CR.

All procedures performed in studies involving human participants were in accordance with the ethical standards of the institutional and/or national research committee(s) and with the Helsinki Declaration (as revised in 2013). All the data in this case report are reported with the informed consent of the patient.

\section{Discussion}

In 2018, the results of Impower133 were first reported by The IASLC World Conference on Lung Cancer (WCLC) and the New England Journal of Medicine simultaneously. It was the first phase III clinical study to confirm that atezolizumab combined with EP chemotherapy is better than EP chemotherapy alone in the treatment of ESSCLC (6), and has therefore rewritten the first-line standard treatment mode for ES-SCLC. In addition, CASPIAN study was another global, open, multicenter phase III study to determine the efficacy and safety of durvalumab \pm tremelimumab combined with platinum-etoposide, followed by durvalumab \pm tremelimumab maintenance therapy compared with platinum-etoposide chemotherapy alone in ES-SCLC patients. The primary end point of the study was OS, and the secondary end points were PFS, ORR, 
safety, and quality of life. In 2019, WCLC published the data of the durvalumab plus platinum-etoposide group and the platinum-etoposide group. The results showed that OS was significantly longer in the durvalumab plus platinumetoposide group than the platinum-etoposide group, with an HR of 0.73 (95\% CI: $0.59-0.91 ; \mathrm{P}=0.0047$ ). The median OS in the durvalumab plus platinum-etoposide group was 13.0 versus 10.3 months in the platinum-etoposide group; the post-hoc 12 -month OS rates were $54 \%$ vs. $40 \%$, and the prespecified 18 -month OS rates were $34 \%$ vs. $25 \%$, respectively. There was no significant difference of incidence of adverse events between two groups, which was consistent with the previous reports (7). Based on the Impower133 and Caspian studies, the United States Food and Drug Administration (FDA) recommend atezolizumab or durvalumab combined with platinum-etoposide as the first-line treatment option for ES-SCLC.

SCLC is a highly invasive neuroendocrine tumor, which has a high degree of malignancy. In the early stage, it can easily metastasize, and in the extensive stage, it develops rapidly. After second-line chemotherapy, SCLC patients always have poor constitution and a performance status score of $\geq 2$. However, the majority of the clinical studies on SCLC have focused on the first-line or second-line treatment schemes, with the PS scores of the selected patients ranging from $0-1$. According to the current relevant clinical research results, patients with SCLC have benefited from anti-PD-1/PD-L1 immunotherapy as a first-line treatment combined with chemotherapy and as a third-line treatment. Despite the preliminary results of CheckMate-032 suggesting that nivolumab + ipilimumab can be used as a second-line treatment option for SCLC (14), exploration of second-line treatment and maintenance treatment in SCLC has generally yielded little success so far. Therefore, a conclusion on the optimal time to introduce immunotherapy as a combination treatment has yet to be reached. In China, most early-stage SCLC patients do not currently receive immunotherapy due to economic reasons or habitual choices in treatment planning. For these patients, there is no standard for deciding the third-line or multi-line treatment, whether to adopt immunotherapy, and which immunosuppressive agents to use.

Programmed cell death 1 ligand 1 (PD-L1), a member of the B7 family, is widely overexpressed in many types of tumors such as lung cancer, breast cancer, glioblastoma, ovarian, and bladder cancers. The engagement of PD- 1 and PD-L1 results in the activation of the inhibitory function of PD-1 in T cells (15). Durvalumab is a selective, high- affinity human IgG1 monoclonal antibody that blocks PDL1 binding to PD-1 and CD80 (8), by inhibiting the activity of inhibitory molecules in the PD-L1 axis, durvalumab release the immune brake of $\mathrm{T}$ cells in the tumor microenvironment, thereby achieve anti-tumor effects.

In the case described above, after multi-line chemotherapy, the patient had poor bone marrow reserve function, anemia, and a WHO performance status score of 2 . Moreover, this case also had a history of chest radiotherapy. Consequently, we had chosen immunotherapy combined with platinum-etoposide chemotherapy (reduced dose) as the third-line treatment. Considering anti-PD-1 antibody may be more likely than anti-PD-L1 antibody to cause immune-related side effects, especially immunerelated pulmonary toxicity in patients with history of chest radiotherapy, we selected durvalumab plus platinumetoposide (reduced dose). However, after the first cycle of treatment, the patient experienced serious side effects such as gastrointestinal bleeding and fatigue. As a result, chemotherapy was cancelled from the second cycle onwards, leaving durvalumab monotherapy. After four cycles, B-ultrasound examination showed that the patient's cervical lesions were completely relieved. Meanwhile, no obvious immune-related side effects, such as rash, diarrhea, fatigue, myelosuppression, and thyroid dysfunction, were encountered. No immune-related pulmonary or hepatorenal toxicities occurred. The last reexamination of the case was in May, 2020: the cervical lesions were in CR, and the lesions in the lung and brain were still stable after more than two years and one year, respectively. It remains to be seen if the patient will achieve pathological remission, and this will need to be confirmed by intervention or operation. This case suggests that immunotherapy can be selected for third-line or multi-line treatment of ES-SCLC, and antiPD-L1 antibody may be the better choice for patients who have poor PS scores.

In conclusion, we reported a case of ES-SCLC who gained CR of local lesions with durvalumab monotherapy as the third-line treatment and experienced no obvious immune-related side effects. Our experience further proves the efficacy and safety of durvalumab, which is consistent with the findings of the CASPIAN study. According to the present research results, immunotherapy in SCLC should be applied in the initial stage; however, most SCLC patients in China do not receive immunotherapy in the early stage. After first-line or second-line treatment, the PS scores of these patients are relatively poor; therefore, it is worth considering flexible application of durvalumab, perhaps as 
a monotherapy or with a dose reduction. This is worthy of further investigation in more clinical trials.

\section{Acknowledgments}

Funding: The research was supported by the Zhejiang Provincial Traditional Chinese Medicine Fund (No. 2019ZB018), Zhejiang Provincial Medical and Health Scientific and Technical Fund (No. 2018KY312), Zhejiang Provincial Medical and Health Scientific and Technical Fund (No. 2020374375) and Zhejiang Provincial Natural and Science Fund (No. LY19H290001).

\section{Footnote}

Reporting Checklist: The authors have completed the CARE reporting checklist. Available at http://dx.doi.org/10.21037/ apm-20-1244

Conflicts of Interest: All authors have completed the ICMJE uniform disclosure form (available at http://dx.doi. org/10.21037/apm-20-1244). The authors have no conflicts of interest to declare.

Ethical Statement: The authors are accountable for all aspects of the work in ensuring that questions related to the accuracy or integrity of any part of the work are appropriately investigated and resolved. All procedures performed in studies involving human participants were in accordance with the ethical standards of the institutional and/or national research committee(s) and with the Helsinki Declaration (as revised in 2013). Written informed consent was obtained from the patient.

Open Access Statement: This is an Open Access article distributed in accordance with the Creative Commons Attribution-NonCommercial-NoDerivs 4.0 International License (CC BY-NC-ND 4.0), which permits the noncommercial replication and distribution of the article with the strict proviso that no changes or edits are made and the original work is properly cited (including links to both the formal publication through the relevant DOI and the license). See: https://creativecommons.org/licenses/by-nc-nd/4.0/.

\section{References}

1. Semenova EA, Nagel R, Berns A. Origins, genetic landscape, and emerging therapies of small cell lung cancer. Genes Dev 2015;29:1447-62.

2. Farago AF, Keane FK. Current standards for clinical management of small cell lung cancer. Transl Lung Cancer Res 2018;7:69-79.

3. Fukuoka M, Furuse K, Saijo N, et al. Randomized trial of cyclophosphamide, doxorubicin, and vincristine versus cisplatin and etoposide versus alternation of these regimens in small-cell lung cancer. J Natl Cancer Inst 1991;83:855-61.

4. Pietanza MC, Byers LA, Minna JD, et al. Small cell lung cancer: will recent progress lead to improved outcomes? Clin Cancer Res. 2015;21:2244-55.

5. Rossi A, Di Maio M, Chiodini P, et al. Carboplatin- or cisplatin-based chemotherapy in first-line treatment of small-cell lung cancer: the COCIS meta-analysis of individual patient data. J Clin Oncol 2012;30:1692-8.

6. Horn L, Mansfield AS, Szcz sna A, et al. First-Line Atezolizumab plus Chemotherapy in Extensive-Stage Small-Cell Lung Cancer. N Engl J Med 2018;379:2220-9.

7. Paz-Ares L, Dvorkin M, Chen $Y$, et al. Durvalumab plus platinum-etoposide versus platinum-etoposide in firstline treatment of extensive-stage small-cell lung cancer (CASPIAN): a randomised, controlled, open-label, phase 3 trial. Lancet 2019;394:1929-39.

8. Stewart R, Morrow M, Hammond SA, et al. Identification and characterization of MEDI4736, an antagonistic antiPD-L1 monoclonal antibody. Cancer Immunol Res 2015;3:1052-62.

9. Antonia SJ, Villegas A, Daniel D, et al. Durvalumab after chemoradiotherapy in stage III non-small-cell lung cancer. N Engl J Med 2017;377:1919-29.

10. Antonia SJ, Villegas A, Daniel D, et al. Overall survival with durvalumab after chemoradiotherapy in stage III NSCLC. N Engl J Med 2018;379:2342-50.

11. Cho DC, Mahipal A, Dowlati A, et al. Safety and clinical activity of durvalumab in combination with tremelimumab in extensive disease small-cell lung cancer (ED-SCLC). Proc Am Soc Clin Oncol 2018;36:abstr 8517.

12. Goldman JW, Dowlati A, Antonia SJ, et al. Safety and antitumor activity of durvalumab monotherapy in patients with pretreated extensive disease small-cell lung cancer (ED-SCLC). Proc Am Soc Clin Oncol 2018;36:abstr 8518.

13. Bondarenko I, Juan-Vidal O, Pajkos G, et al. Preliminary efficacy of durvalumab plus tremelimumab in platinumrefractory/resistant ED-SCLC from arm A of the phase II BALTIC study. Ann Oncol 2018;29:viii596-602.

14. Hellmann MD, Ott PA, Zugazagoitia J, et al. Nivolumab(nivo) \pm ipilimumab(ipi)in advanced small- 
cell lung cancer(SCLC):first report of a randomized expansion cohort from CheckMate 032. J Clin Oncol 2017;35:abstr 8503.

15. Sun C, Mezzadra R, Schumacher TN. Regulation and Function of the PD-L1 Checkpoint. Immunity 2018;48:434-52.

(English Language Editor: J. Reynolds)

Cite this article as: Zhou Q, Zhao J, Wang J, Bao G, Gong LY. Durvalumab monotherapy as a third-line treatment for extensive-stage small-cell lung cancer: a case report. Ann Palliat Med 2020;9(4):2386-2392. doi: 10.21037/apm-20-1244 